Bem. r. 5 Sekunden nach dem beobachteten Eintritte bemerkte ich Wolken, welche vielleicht schon früher vorhanden waren. - 2. u. 3. Die Sterne waren sehr schwach, die Beobachtung war schwierig und nicht ganz sicher.

Die Nummern der Sterne sind dem Kataloge: "Resultados del Observatorio Nacional Argentino. Vol. z2, Cordoba I 9 I 3 " entnommen.

Krakau, I 9 I 7 Sept. 2.

W. Dsiewulski.

Beobachtungen auf der Universitätssternw. Leipzig.

Zur Beobachtung wurde der Schrödersche Kometensucher von $14 \mathrm{~cm}$ Öffnung mit Ioo-facher Vergrößerung und ein lichtstarkes Prismenfernrohr von 8-facher.Vergr. benutzt. $\mathrm{Da}$ eine erfolgreiche Beobachtung von Sternbedeckungen mit den vorhandenen Hilfsmitteln nicht zu erwarten war, so habe ich mich darauf beschränkt, die Färbung und die Helligkeitsverteilung $\mathrm{zu}$ verfolgen.

Nachdem sich der Mond ganz über die Dunstschicht am Horizont erhoben hat, ist ein der scharfen Schattenlinie vorangehendes Band schwächeren Schattens von ungefähr I' Breite deutlich zu erkennen.

$8^{\mathrm{h}} 49^{\mathrm{m}} \cdot 6$ (alle Zeitangaben in m. Z. Greenw.) helle Linie an dem, dem Mare Crisium benachbarten Randpunkt noch zu sehen, Ostrand unsichtbar. Beginn der Totalität.

Während der Totalität zeigte sich im allgemeinen folgender Verlauf der Färbung: Die Mitte der sich von $30^{\circ}$ allmählich auf ca. $80^{\circ}$ des Umfanges ausdehnenden zartgelben Sichel verschob sich fast gleichmäßig von einem dem Mare Crisium benachbarten Randpunkt, wo sie $8^{\mathrm{h}} 57^{\mathrm{m}}$ verzeichnet wurde, bis zu einem dem Kap Heraclides benachbarten Randpunkt, wo sie sich $9^{\mathrm{h}} 59^{\mathrm{m}}$ befand. Die Mitte der dunkelsten Schattenpartie wurde $8^{\mathrm{h}} 57^{\mathrm{m}}$ beim Krater Agatharchides, $9^{\mathrm{h}} 27^{\mathrm{m}}$ am Südrande des Ptolemäus, $9^{\mathrm{h}} 3^{6^{\mathrm{m}}}$ bei Delambre verzeichnet. Gleichzeitig verschiebt sich eine schwach rötliche Sichel rom West- zum Südpunkt des Randes. Vom Rande nach dem Innern der Mondscheibe $z u$ ist die Färbung rötlich bis rostbraun; hier sind Einzelheiten der Oberfläche noch zu erkennen, was in dem schwarzbraun gefärbten, dunkelsten Gebiet, dessen Ausdehnung der des Mare Imbrium gleich geschätzt wurde, nicht möglich war. $10^{\mathrm{h}} \mathrm{I} 9^{\mathrm{m}}$, das dunkle Gebiet hat sich bis an den Westrand geschoben; dieser ist weder im Kometensucher noch im Prismenglas zu erkennen. I $\mathrm{o}^{\mathrm{h}} 27^{\mathrm{m}} \cdot 3$ hellste Stelle in der Umgebung der Hercynischen Berge. Ende der Totalität. I I $^{\mathrm{h}} 26^{\mathrm{m}} \cdot 8$ keine Schattenspur mehr zu erkennen.

Die Zeitangaben sind wegen der erwähnten Verwaschenheit der Schattenlinie und infolge anderer Fehlerquellen schätzungsweise mit einer Ungenauigkeit von $0^{\mathrm{m}} \cdot 5$ behaftet.

Bei dieser Gelegenheit möchte ich auf die interessanten Auseinandersetzungen Keplers (Kepleri opera. Editio Frisch Bd. 2, S. 297-308) über die Erklärung des ganzen Vorganges, auf die mich Prof. Bruns aufmerksam gemacht hat, hinweisen, da diese Stelle selbst in Fachkreisen nur wenig bekannt sein dürfte.

$$
\text { Leipzig, r } 9 \text { I } 7 \text { Juli I } 4 \text {. }
$$

F. Weber.

Beobachtungen auf dem Observatorium

der k. k. Technischen Hochschule in Lemberg.

Hier wurde die Mondfinsternis von mir an einem parallaktischen Refraktor von Fraunhofer-Ressel von $122 \mathrm{~mm}$ Öfnung und $162 \mathrm{~cm}$ Brennweite unter Anwendung I 20 -facher Vergrößerung, sowie von dem Assistenten $\mathcal{F}_{\text {. Dzierzynski an }}$ einem Fraunhoferschen Fernrohr von $72 \mathrm{~mm}$ Öffn. und $\mathrm{I}$ I $5 \mathrm{~cm}$ Brennweite mit 85 -facher Vergr. beobachtet. An beiden Instrumenten sind sämtliche vier Kontakte des Mondrandes mit der Schattengrenze beobachtet worden; an dem ersteren außerdem die Ein- und Austritte einiger Mondkrater.

Das Wetter war die ganze Zeit hindurch sehr günstig; nur gegen Ende der Erscheinung war der Mond einige Minuten etwas verschleiert durch einen leichten Cirrus, welcher die ohnehin verschwommene Schattengrenze noch verschwommener erscheinen ließ, sich aber noch vor dem IV. Kontakt wieder verzog.

Im Beobachtungsprotokoll sind die Zeiten auf einzelne Sekunden notiert; da jedoch bei der Beobachtung das Gefühl bestand, daß dieselben jedenfalls auf mehrere Sekunden (die Kontaktbeobachtungen noch mehr) unsicher sind, habe ich sie nachträglich, nach Anbringung der Uhrkorrektionen und der Reduktion auf m.Z. Greenw., auf Zehntelminuten abgerundet.

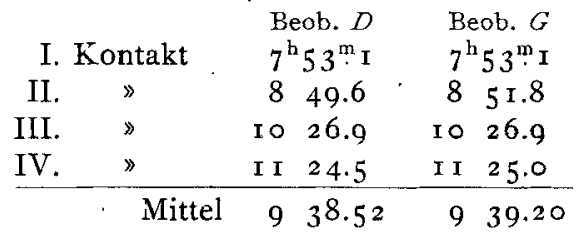

Das Mittel erscheint somit nach Beobachter $D$ um $0^{\text {m }} 33$ verfrüht, nach $G$ um $\circ^{\mathrm{m}} \cdot 35$ verspätet gegenüber der Ephemeride des Berl. Astron. Jahrbuchs.

Ferner wurden beobachtet:

Eintritte (Beob, G) Austritte (Beob. G)

Grimaldi (Mitte) $\quad 7^{\mathrm{h}} 5^{6^{\mathrm{m}} \cdot 7}$ Riccioli $\quad 10^{\mathrm{h}} 30^{\mathrm{m}} \cdot 2$

Hevelius (?) $\quad$\begin{tabular}{ll|ll}
7 & 57.3 & Aristarchus & 10 34.7
\end{tabular}

\begin{tabular}{lrr|ll} 
Aristarchus & 8 & 3.1 & Gay-Lussac & ro 45.5
\end{tabular}

Copernicus (Mitte) 8 I 2.7 Copernicus (Mitte) ro 47.5

\begin{tabular}{lll|lll} 
Tycho (Mitte) & 8 & I 9.3 & Tycho (Mitte) & 10 & 57.6
\end{tabular}

$\begin{array}{llllll}\text { Menelaus } & 8 & 31.0 & \text { Menelaus } & \text { I I } & 2.7 \text { (Cirri) }\end{array}$

\begin{tabular}{lll|lll} 
Dionysius & 8 & $\mathbf{3} \mathbf{1 . 2}$ & Proclus & I I & I 3.7
\end{tabular}

Proclus

8 \begin{tabular}{ll}
31.2 \\
\hline
\end{tabular}

Der Schatten hatte die gewöhnliche rote Färbung (in der Nähe des Randes etwas mehr orange); er war ziemlich hell, sodaß auch innerhalb des Schattens die Details der Mondoberfläche größtenteils deutlich sichtbar waren.
Lemberg, I9r7 Aug. 2.

\title{
Resultate aus den Babelsberger Beobachtungen von Sternbedeckungen während der Monfinsternis vom 4. Juli 1917. Von E. Bernewitz.
}

Von den beobachteten $\mathrm{I}_{3}$ Eintritten und 5 Austritten konnten nur 8 zur Bestimmung der Korrektion des Mondortes und Mondhalbmessers herangezogen werden, weil nur

für diese Anzahl von Sternen genügend genaue Positionen vorhanden waren. Andererseits mußten zwei Beobachtungen verworfen werden, da offenbar die Zeiten für die Austritte 
der Sterne 1 3166 und 13218 des Cordobaer Kataloges unsicher sind. Stern 13166 ist ein Doppelstern, während Stern I 32 I 8 sehr streifend am bereits wieder voll erleuchteten Mondrande austrat.

Als Grundlage für die Rechnung dienten die Monđörter des N. A. rgI7. Für die Horizontal-Äquatorealparallaxe des Mondes ist nach dem N. A. der Wert $57^{\prime} 2^{\prime \prime} \circ 09$, für den Halbmesser des Mondes nach Oudemans 0.27264 angenommen worden. Die Sternörter sind der AG-Zone Cordoba ent- nommen, sie sind schon im Eingang dieser Nummer angegeben.

Die Rechnung wurde nach den von Chauvenet in seinem Manual of Spherical and Practical Astronomy, Vol. I pag. $55^{\circ}$ gegebenen Formeln ausgeführt. Die Koordinaten der Babelsberger Sternwarte (großer Refraktor) sind:
Länge

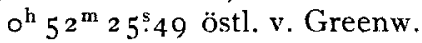
Breite $+52^{\circ} 24^{\prime} 24^{\prime \prime} .2$

Seehöhe ca. $80 \mathrm{~m}$.

$$
\text { Mịt diesen Daten ergeben sich die nachstehenden Elemente der Bedeckung in der Bezeichnung von Chauvenet: }
$$

\begin{tabular}{|c|c|c|c|c|c|c|c|c|}
\hline & $13168(\mathrm{~A})$ & $13167(\mathrm{~A})$ & $13197(\mathrm{E})$ & $13209(E)$ & I32 I I $(E)$ & I 32 I $8(E)$ & $13226(E)$ & $1321 \mathrm{I}(\mathrm{A})$ \\
\hline$T_{0}$ & 9.0 & 9.0 & 9.25 & 10.0 & 10.0 & 10.5 & 10.75 & I I.O \\
\hline & -0.161337 & -o.1 49979 & $-0.5^{6} 5^{195}$ & -0.336722 & -0.38 I I7 9 & -0.208033 & -0.247023 & +0.219534 \\
\hline & -0.323652 & -0.3186 18 & -0.300065 & -0.176706 & - o.I 74 I 77 & -0 & -0. & -0.05 \\
\hline & + I.rI64I3 & +1.080120 & +1.027736 & +0.685 I87 & +0.698364 & +0.6 & + I.I 89532 & +0.812710 \\
\hline & +0.931318 & +0.932476 & +0.936652 & +0.956248 & +0.956600 & $+0.962 \times 86$ & +0.966405 & +0.965770 \\
\hline & $79^{\circ} 34^{\prime} 53^{\prime \prime} 3$ & $79^{\circ} 35^{\prime} 7^{\prime \prime} \cdot 5$ & $79^{\circ} 20^{\prime} 23^{\prime \prime} \mathrm{I}$ & $79^{\circ}$ 工 $4^{\prime}$ I $3^{\prime \prime} .9$ & $79^{\circ}$ I $3^{\prime} \quad 8 . \prime \circ$ & $79^{\circ} 10^{\prime} \quad 0^{\prime \prime} 8$ & $79^{\circ} 6^{\prime} \quad 8^{\prime \prime} .7$ & $79^{\circ} \mathrm{I} 3^{\prime} 4 I^{\prime \prime} .1$ \\
\hline & $\begin{array}{lll}-34 & 3 & 31.9\end{array}$ & $\begin{array}{lll}-24 & 53 & 6.4\end{array}$ & $\begin{array}{lll}210 & 32 & 39.4\end{array}$ & I I $952: 29.2$ & I27 $57 \quad 40.6$ & $\begin{array}{lll}\text { I } 6 & 30 & 33.6\end{array}$ & $\begin{array}{lll}247 & 5 & \text { I } 8.8\end{array}$ & $\begin{array}{lll}47 & 34 & 24.7\end{array}$ \\
\hline & $+193^{\mathrm{s}} 5^{\circ}$ & $+323^{5} \cdot 53$ & $+5^{2} \cdot 54$ & $+425^{\mathrm{s}} \mathrm{I} 5$ & $+495^{5} \cdot 5$ & +124.96 & $+106: 16$ & $-324^{\mathrm{s}} \mathrm{I} 9$ \\
\hline$-N$ & $-21.8 z$ & -21.75 & $-2 \mathrm{r} .48$ & -20.93 & -20.34 & $-\quad 18.17$ & -34.53 & -25.05 \\
\hline
\end{tabular}

Mit diesen Größen wurden die Fehlergleichungen abgeleitet:

$$
\begin{aligned}
& -21.82=-1.633 \cdot \gamma-1.104 \cdot \gamma+1.972 \cdot \pi d k \\
& -21.75=-1.633-0.758+1.801 \\
& -21.48=-1.633+0.963-1.896 \\
& -20.93=-1.633-2.841-3.277 \\
& -20.34=-1.633-2.092-1.653 \\
& -18.17=-1.633-3.272-3.656 \\
& -34.53=-1.633+3.866-4.191 \\
& -25.05=-1.633+1.785+2.418
\end{aligned}
$$

In ihnen steht links die Differenz: beobachtete - berechnete Eintritts- resp. Austrittszeit; rechts ist $\boldsymbol{\pi} \boldsymbol{1} k$ die Verbesserung des angenommenen Mondhalbmessers, während $\gamma$ und $\vartheta$ die folgenden Kombinationen der Ephemeridenkorrektionen sind:

$$
\begin{gathered}
\gamma=\sin N \cos \delta \cdot \boldsymbol{I}\left(\alpha-\alpha^{\prime}\right)+\cos N \cdot \boldsymbol{I}\left(\delta-\delta^{\prime}\right) \\
\vartheta=-\cos N \cos \delta \cdot \boldsymbol{I}\left(\alpha-\alpha^{\prime}\right)+\sin N \cdot \alpha\left(\delta-\delta^{\prime}\right) \\
\text { Neubabelsberg, I9 I } 7 \text { Juli } 3 \text { I. }
\end{gathered}
$$

Die Koeffizienten sind so gewählt, daß sich $\gamma$ und $\boldsymbol{y}$ in Bogensekunden ergeben. Die Auflösung lieferte nachstehende Werte für die drei Unbekannten :

$$
\begin{aligned}
& \gamma=+\mathrm{I} 4.26 \quad y=-\mathrm{x} \text { ".85 } \quad \pi d k=+0.44 \\
& \boldsymbol{A}\left(\alpha-\alpha^{\prime}\right)=+\mathrm{I} 5^{\prime \prime} .55 \quad \boldsymbol{A}\left(\delta-\delta^{\prime}\right)=+0.8 .3 .
\end{aligned}
$$

Aus den übrigbleibenden Resten folgt der mittl. Fehler einer Eintritts- oder Austrittszeit $z u+2 \mathrm{~s} .66$; hieraus wurden die nachstehend aufgeführten genäherten mittleren Fehler der Unbekannten berechnet:

$\varepsilon(A \alpha)= \pm 0.67 \quad \varepsilon(\mathcal{A} \delta)= \pm 0.49 \quad \varepsilon(\pi / k)= \pm 0.33$ Nimmt man an, daß sich die Fehler der Sternörter in ihrem Einfluß auf das Resultat im Mittel zerstören, so folgt schließlich :

$\mathcal{A} \alpha_{\mathbb{C}}=+{ }_{1}^{5.04} \pm 0.05 \quad d d_{\mathbb{C}}=+0.8 \pm 0.5 \quad k=0.27276$.

E. Bernewitz.

\section{Mikrometrische Beobachtungen der Mondfinsternis 1917 Juli 4. Von C. Wirtz.}

Beobachtungsort Charlottenburg: $\varphi=+5^{\circ} 30^{\circ} \mathrm{I} 83_{3}^{\prime \prime}, \lambda=0^{\mathrm{h}} 53^{\mathrm{m}} 21^{\mathrm{s}} \cdot 35$ östl. von Greenw.

I. Während der totalen Mondfinsternis I 9 I 7 Juli 4 habe ich mit allerdings recht geringen optischen Hilfsmitteln mikrometrische Beobachtungen des Erdschattens angestellt, deren überwiegender Teil die scheinbare Vergrößerung des Erdschattens mit befriedigender Endgenauigkeit ergibt, während im andern Teil der Messungen ein systematischer Gang erscheint, der nach seiner absoluten Größe und Regelmäßigkeit vielleicht Erwähnung verdient.

Als Instrument diente ein Mikrometerfernrohr mit zerschnittenem Objektiv. Es ist eine einfache Heliometerkonstruktion der Firma Bamberg, wie sie in der Kaiserlichen Marine als Distanzmesser nach Schaub bezeichnet und in dem "Handbuch der nautischen Instrumente «, 2. Aufl., Berlin I 890, S. 354 beschrieben wird. Das Fernrohr hat nur $33 \mathrm{~mm}$ Objektivdurchmesser, $37 \mathrm{~cm}$ Brennweite und eine 20 fache Vergrößerung. Jede Objektivhälfte weist daher nur die Lichtstärke eines vollständigen Objektivs von $23 \mathrm{~mm}$ auf. Ein Stativ gehört nicht $z u$ dem Apparat, und die Messungen müssen aus freier Hand unter Aufstützen des haltenden Armes vonstatten gehen.

Die Schraubenrevolution $R$ wurde im Laufe der abendlichen Beobachtungen aus 7 vollständigen Messungen des vertikalen und horizontalen Monddurchmessers, mit je 4 Einstellungen, abgeleitet zu $R=25_{2}^{2}$. 4 . Der Wert gehört also zu der während der Beobachtungen innegehaltenen Fokalstellung, die in Ermangelung einer Fokalskala sich nicht näher fixieren läßt. Der Abstand $\ell$ der Objektivhälften stellte sich zwar als recht beträchtlich heraus, doch wirkte er bei der Größe der gemessenen Distanzen noch nicht störend. Aus Beobachtungen an Sternen wurde diese Distanz gefunden zu $e=5^{2 \prime}$.

Die Finsternisbeobachtungen selbst bestanden aus 37 Messungen der Länge der dem Schatten und der Mondscheibe gemeinsamen Sehne und aus i 6 Einstellungen der Breite der hellen Mondsichel. Die Reihe zerfällt in Gruppen von je 4 